\title{
7T MRI for neurodegenerative dementias in vivo: a systematic review of the literature
}

\author{
Elizabeth Frances McKiernan, John Tiernan O'Brien
}

Department of Psychiatry, Cambridge Biomedical Campus, University of Cambridge, Cambridge, UK

\section{Correspondence to}

Dr Elizabeth Frances McKiernan, Department of Psychiatry, School of Clinical Medicine, Cambridge Biomedical Campus, University of Cambridge, Box 189, Level E4, Cambridge CB2 OSP, UK; em654@medschl.cam. ac.uk

Received 4 October 2016 Revised 29 December 2016 Accepted 9 January 2017 Published Online First 4 March 2017

CrossMark

To cite: McKiernan $\mathrm{EF}$, O'Brien JT. J Neurol Neurosurg Psychiatry 2017:88:564-574.

\section{ABSTRACT}

The spatial resolution of 7T MRI approaches the scale of pathologies of interest in degenerative brain diseases, such as amyloid plaques and changes in cortical layers and subcortical nuclei. It may reveal new information about neurodegenerative dementias, although challenges may include increased artefact production and more adverse effects. We performed a systematic review of papers investigating Alzheimer's disease (AD), Lewy body dementia (LBD), frontotemporal dementia (FTD) and Huntington's disease (HD) in vivo using 7T MRI. Of 19 studies identified, 15 investigated AD (the majority of which examined hippocampal subfield changes), and 4 investigated HD. Ultrahigh resolution revealed changes not visible using lower field strengths, such as hippocampal subfield atrophy in mild cognitive impairment. Increased sensitivity to susceptibilityenhanced iron imaging, facilitating amyloid and microbleed examination; for example, higher microbleed prevalence was found in AD than previously recognised. Theoretical difficulties regarding image acquisition and scan tolerance were not reported as problematic. Study limitations included small subject groups, a lack of studies investigating LBD and FTD and an absence of longitudinal data. In vivo 7T MRI may illuminate disease processes and reveal new biomarkers and therapeutic targets. Evidence from $A D$ and $H D$ studies suggest that other neurodegenerative dementias would also benefit from imaging at ultrahigh resolution.

\section{INTRODUCTION}

Neurodegenerative dementias, including Alzheimer's disease (AD), Lewy body dementia (LBD), frontotemporal dementia (FTD) and the dementia of Huntington's disease (HD), account for the majority of dementia syndromes diagnosed worldwide. ${ }^{1} \mathrm{AD}$ produces a syndrome characterised by deficits in multiple cognitive areas, including orientation and social function; most often, the predominant symptom is of progressive anterograde amnesia. ${ }^{2}$ Diagnostic hallmarks of $\mathrm{AD}$ are amyloid plaques and neurofibrillary tangles found at postmortem, which typically follow a regional path. $^{3}$ A number of in vivo biomarkers have been described, and current recommended criteria for diagnosis of probable $\mathrm{AD}$ include neuroimaging markers of abnormal brain amyloid deposition and neuronal degeneration. ${ }^{4}$ Amyloid deposition can be imaged via increased uptake of amyloid markers on positron emission tomography (PET), such as 11[C]PIB. ${ }^{5}$ Neuronal degeneration in AD can be imaged via decreased uptake of [18]F-FDG PET (an indicator of tissue metabolism) and via structural MRI, which shows typical patterns of atrophy in the temporal and parietal lobes. ${ }^{6}$

Features of LBD include the triad of fluctuating cognition, visual hallucinations and extrapyramidal movement disorder ${ }^{7}$ and are characterised by the presence of abnormal alpha-synuclein in neurons and glial cells found in the brainstem, limbic system and cortex at postmortem. ${ }^{8}$ The most commonly used neuroimaging technique in the diagnosis of LBD is dopaminergic imaging of the basal ganglia with sensitivity of $87 \%$ and specificity of $94 \%$ for differentiating between LBD/Parkinson's disease dementia (PDD) and other dementia types. ${ }^{9}$ A recent review of LBD imaging described changes identified using MRI, including volumetric differences in the substantia innominate and putamen and degeneration of the pons and thalamus. ${ }^{10}$

In FTD, episodic memory is relatively preserved; changes in personality, disinhibition and language difficulties predominate. It is characterised by atrophy of frontal and temporal lobes and the histological presence of TAR DNA-binding protein 43 (TDP-43) and/or fused in sarcoma (FUS) pathology and may mimic atypical AD clinically. ${ }^{11} \mathrm{~A}$ number of in vivo neuroimaging markers have been described, including atrophy in frontal insula, cingulate, striatum and anterior temporal lobe on structural MRI, frontal lobe hypoperfusion using arterial spin labelled (ASL) perfusion imaging and reduced fractional anisotropy (FA) in frontal regions using diffusion tensor imaging (DTI). ${ }^{11}$

HD is an autosomal-dominant trinucleotide repeat disorder with an estimated prevalence of 5.7 per 100000 in a majority of Caucasian populations (in whom incidence is highest). ${ }^{12}$ Characterised by the presence of cerebral intranuclear inclusions of mutated Huntingtin, it produces movement disorder, cognitive decline and behavioural symptoms. Dementia can occur at any point in the disease process, and cognitive deficits are primarily in concentration, short-term memory and executive function. Carriers are identified via genetic testing, which shows an expansion of CAG trinucleotide repeats within the Huntingtin gene on chromosome $4 .{ }^{13} \mathrm{~A}$ reduction in striatal volume is the most consistent MRI finding in HD and correlates with age at disease onset, duration of disease and CAG repeat length. Changes in caudate and putamen volumes, cortical thinning and white matter (WM) atrophy have also been identified. ${ }^{14} \mathrm{~A}$ large longitudinal multicenter trial used 3T MRI to demonstrate progressive grey matter and WM, whole brain and regional cortical atrophy in HD and premanifest 
(prodromal or premotor) HD, with the largest effect sizes seen in the caudate and WM. ${ }^{15}$

MRI is an important clinical tool used to rule out reversible causes of dementia and to aid in the diagnosis of early dementias or those presenting in an atypical manner. ${ }^{16}$ Neuroimaging dementia research is largely concerned with the discovery of new biomarkers with such aims as earlier diagnosis, identification of 'at-risk' individuals and those with 'prodromal' dementia syndromes, monitoring of disease progression and identification of new treatment targets by illuminating the natural history of the disease in vivo. Increasing static field strength $\left(\mathrm{B}_{0}\right)$ from 1.5 $\mathrm{T}$ to $3 \mathrm{~T}$ has allowed imaging of macro and microstructures in the brain, for example, analysis of whole hippocampal volumes and differentiation between hippocampal subfield volumes in health and disease. ${ }^{17}$ However, even at 3T, MRI voxels typically measure millimetres. Consequently, the resulting images remain several orders of magnitude away from the size of pathologies of interest; cortical layers, hippocampal subfields, brainstem nuclei, plaques, tangles and microbleeds are typically measured in micrometres. Seven tesla MRI may allow increases in image resolution, allowing us to visualise these microstructures and pathology in vivo.

Seven tesla MRI creates both opportunities and challenges for the neuroscience community (see the work of Balchandani and Naidich ${ }^{18}$ for a technological summary of 7T MRI capabilities). In theory, image resolution increases by around $30 \%$ as $\mathrm{B}_{0}$ increases from $3 \mathrm{~T}$ to $7 \mathrm{~T}$. The relationship between image resolution and $\mathrm{B}_{0}$ is complex, because resolution is determined by a number of factors, including signal-to-noise ratio (SNR), voxel size and image acquisition time. Theoretically, SNR increases linearly with increasing $\mathrm{B}_{0}$ and should more than double as $\mathrm{B}_{0}$ increases from $3 \mathrm{~T}$ to $7 \mathrm{~T}$. However, in practice, maintenance of field homogeneity becomes more challenging; spatial and intensity distortions occur more commonly as $\mathrm{B}_{0}$ increases. Contrast between tissue types also determines image resolution and is influenced by such factors as $\mathrm{T} 1, \mathrm{~T} 2$ and $\mathrm{T} 2 *$ relaxation times and sensitivity to susceptibility (phase difference between tissues).

Tissue relaxation values change as $\mathrm{B}_{0}$ increases-slower and converging for T1 and faster for T2 and T2*. Faster T2* results in better contrast of iron deposits, calcifications and deoxyhaemoglobin; however, drop-out and susceptibility artefacts increase, particularly in brain areas close to bone, vasculature or cerebrospinal fluid. Sensitivity to susceptibility can be used to measure paramagnetic substances such as iron and increases in proportion to increasing $\mathrm{B}_{0}$ and longer echo time (TE). Increasing $\mathrm{B}_{0}$ allows shorter TEs to be used, producing potential improvements in image resolution and shorter acquisition times. Acquisition times are important in dementia imaging, as participants may find long scan durations particularly difficult to tolerate, and many dementia processes are accompanied by movement disorders. Image acquisition at $7 \mathrm{~T}$ may require a choice between ultrahigh resolution, which is disproportionately adversely affected by head and physiological movement, and faster acquisition times, which may ameliorate the effects of excess motion. $^{19}$

Newly developed technologies and imaging protocols can overcome some of the challenges previously described; for example, advanced $\mathrm{B}_{0}$ shimming and small voxel volumes have been found to compensate for increased field inhomogeneity. New radio frequency (RF) pulse sequences are also required, because as $\mathrm{B}_{0}$ increases to $7 \mathrm{~T}$, the wavelength of the RF is close to the diameter of the head, leading to signal drop-out and contrast changes in brain peripheries. ${ }^{18}$ Areas of interest in dementia research include small peripheral brain regions such as the hippocampus, entorhinal cortex (ERC) and amygdala, areas identified to play a large role in postmortem histological studies. ${ }^{20}$ Due to their small size and complex internal structures, such regions are likely to benefit from higher resolution imaging; however, they are also brain most difficult to examine using MRI due to artefact production.

In humans, potential adverse effects produced by high electromagnetic fields used in MRI include transient effects such as vertigo, peripheral nerve stimulation, metallic taste in the mouth and impaired cognition. More serious longer term effects include damage to implanted medical devices such as pacemakers, intracranial clips and neurostimulators, which may be damaged directly by the applied magnetic fields or by torque, which may displace devices within the body. ${ }^{21}$ There are limits to the amount of RF power deposition (specific absorption rate (SAR)) that can be applied in human subjects. SAR increases proportional to $\mathrm{B}_{0}{ }^{2}$. This large increase in SAR between 3T and $7 \mathrm{~T}$ means that the number, duration and amplitude of RF pulses that can be applied are restricted due to heating effects on objects in the scanner bore. These effects may be especially problematic for patient populations being imaged for dementia research, as thermoregulation may be impaired in people who are older, have health problems or take medications. ${ }^{22}$

The aim of this literature review is to systematically describe and evaluate published studies that have used 7T MRI in vivo in subjects with neurodegenerative dementias. Gaps in the current literature and potential future applications for this technology in neurodegenerative dementias will then be discussed.

\section{METHODS}

The initial search was completed on PubMed using the following terms: '7T' OR '7 Tesla' OR 'seven tesla' OR 'ultrahigh resolution' AND 'MR' OR 'MRI' OR 'magnetic resonance imaging' AND 'neurodegen"' OR 'Mild cognitive impairment' OR 'MCI' OR 'Alzheimer's' OR 'AD' OR 'Lewy body dementia' OR 'LBD' OR 'Parkinson's disease dementia' OR 'PDD' OR 'Frontotemporal dementia' OR 'FTD' OR 'Huntington*' OR 'HD'. Papers published between 1 January 1985 and 1 December 2016 were searched. These search criteria produced 393 results. Articles reviewed were limited to those related to in vivo human studies, and only papers in English were included. Relevant papers were identified by manual review of titles and abstracts. Sixteen relevant studies were identified. The reference lists of these papers were searched to identify further relevant papers, and 3 further relevant studies were identified, yielding a total of 19 papers (figure 1).

\section{RESULTS}

\section{Papers on AD}

Papers identified fell broadly into four groups: those reporting medial temporal lobe structures, those reporting vascular changes (microbleeds and microinfarcts), those investigating markers of amyloid and other papers. In all papers, $\mathrm{AD}$ was defined according to the NINCDS-ADRDA (National Institute of Neurological and Communicative Disorders and Stroke-Alzheimer's Disease and Related Disorders Association) criteria, ${ }^{24}$ and in studies with a mild cognitive impairment (MCI) group, this was defined according to either the NIA-AA (National Institute on Aging-Alzheimer's Association) criteria $^{23}$ or Petersen criteria. ${ }^{24}$ Additional criteria, such as minimum Mini-Mental State Examination (MMSE) score and an adequate level of English comprehension, were stipulated in some studies. 


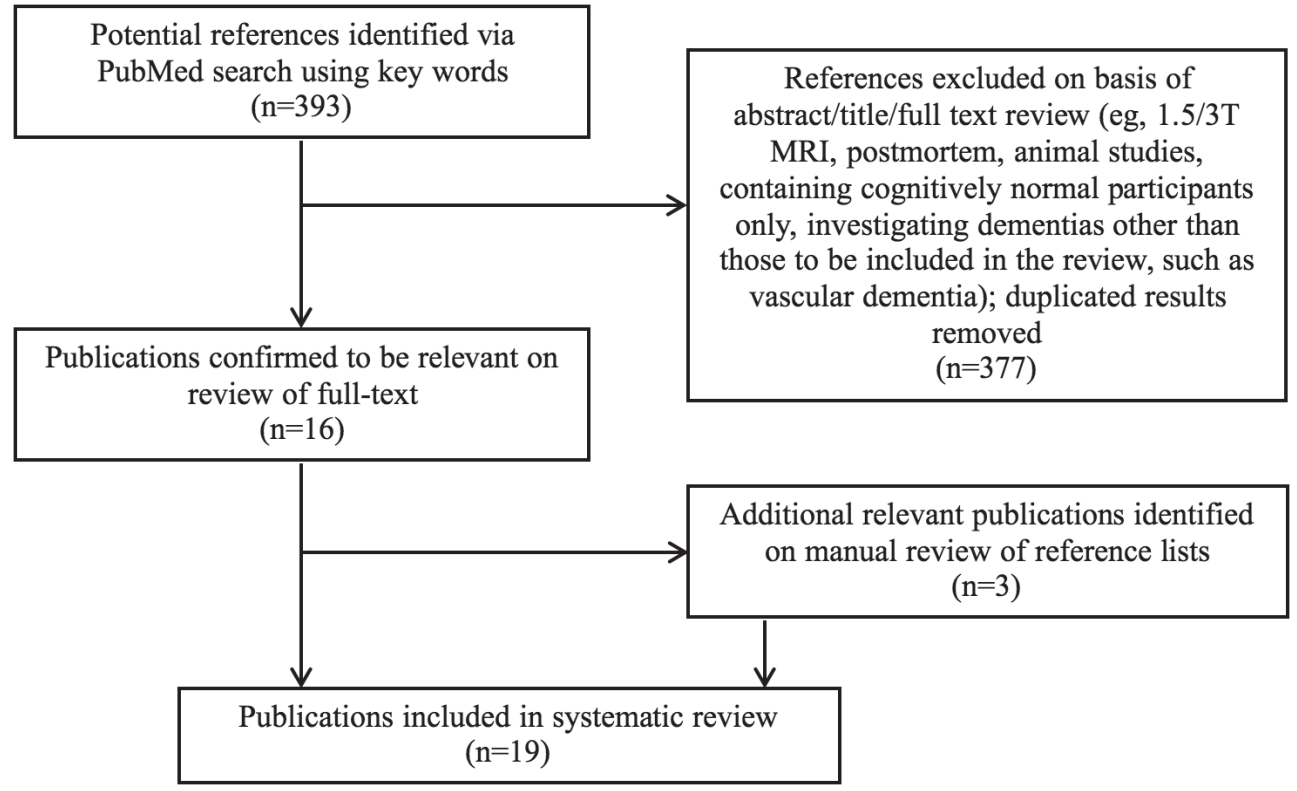

Figure 1 Flow diagram depicting the systematic review process.

Seven in vivo 7T MRI studies including individuals with AD and/or MCI examining hippocampal volumes and subfields were identified (table 1). Image acquisition methods were similar across the majority of the studies, and T2-weighted fast/turbo spin echo acquisition sequences were mostly used. Reported in-plane resolutions varied between 0.166 and $0.90 \mathrm{~mm}^{2}$. Segmentation of the hippocampus and its subfields was mostly performed manually based on anatomical landmarks. No papers presented a fully automated approach for subfield segmentation. Papers used various anatomical landmarks for segmentation, and differences in approach may explain some current and future variability between findings at 7T. There was consensus between these papers that individual hippocampal subfields can be differentiated using 7T MRI in vivo, and the morphometry of these subfields is altered in AD and may also be altered in MCI. There was no consensus within the literature regarding which layers are most affected and in what ways; the most commonly reported area affected in $\mathrm{AD}$ was the cornu ammonis layer 1 (CA1), specifically the strata radiatum, lacunosum and moleculare (CA1-SRLM). Volume reductions in this subfield were seen consistently in early $\mathrm{AD}$, and correlations between CA1 width and delayed recall memory performance were found. ${ }^{25-28}$

Eight other in vivo 7T MRI studies including individuals with $\mathrm{AD}$ and/or MCI were identified (table 2). Four papers investigated amyloid plaque deposition, one paper investigated microbleed prevalence, one paper investigated microinfarct prevalence, one paper investigated perivascular density and one paper addressed movement artefact reduction within the MRI scanner. The studies investigating amyloid plaque deposition used T2*-weighted gradient echo (GRE) acquisition sequences. Reported in-plane resolutions varied between 0.156 and $0.5 \mathrm{~mm}^{2}$. At such resolutions, it is not possible to visualise the majority of amyloid plaques directly, their mean diameter being in the tens of micrometres. ${ }^{29}$ Various methods were used to image amyloid, and susceptibility-weighted imaging (SWI) was used to directly visualise 'senile-plaque-like-pathology', small hypointense areas in the parietal cortex, ${ }^{30}$ a finding that was not replicated elsewhere. ${ }^{31}$ Increased phase shift (seen in the presence of iron, which colocalises with amyloid) was found in $\mathrm{AD}$ throughout the cortex although not in the hippocampi ${ }^{31}{ }^{32}$; in addition, this effect was found to be greater in early-onset $\mathrm{AD}$ than late-onset $\mathrm{AD} .{ }^{32}$ Raised quantitative susceptibility mapping (QSM) values (used as a proxy for cortical iron burden) were found to colocalise with amyloid beta $(\mathrm{A} \beta)$-plaque-load (measured using C[11]-PiB-PET) in apolipoprotein E (APOE) e4-positive MCI but not in the APOE e4-negative MCI or in APOE e4-positive controls. ${ }^{33}$

Only one paper directly compared results obtained using 7T and $3 \mathrm{~T} \mathrm{MRI}^{34}$; statistically significant differences in microbleed prevalence were seen between controls and AD/MCI using 7T only.

Only two papers commented on tolerability of the scan and adverse effects ${ }^{25}{ }^{35}$ : one reported a single case of tinnitus, ${ }^{35}$ and the other reported no significant adverse effects. ${ }^{25}$

\section{Papers on HD}

Four in vivo 7T MRI studies including individuals with HD and/or premanifest HD were identified (table 3). Definitions of HD and premanifest HD were not consistent within these papers. In all cases, patients were positive for HD mutation with $\geq 40$ CAG repeats; however, divisions between premanifest HD and HD were defined according to Unified Huntington's Disease Rating Scale motor score, ${ }^{36}$ and various cut-off values were chosen.

Two papers measured iron deposition in basal ganglia structures, one paper investigated changes in the blood-brain barrier, and one paper reported on a quantitative textural analysis. Similar to the AD amyloid-deposition papers previously described, the HD papers investigating iron deposition used T2*-weighted GRE acquisition sequences and reported in-plane resolutions of 0.5 and $1 \mathrm{~mm}^{2}$. Image susceptibility increases in the caudate nucleus (suggesting greater iron deposition) were reported in premanifest $\mathrm{HD},{ }^{37} 38$ and were found to correlate with the CAG-Age Product Scaled (CAP) score ${ }^{39}$ (used to estimate the probability of progression to clinical HD within 5 years).

One paper commented on scan tolerability and adverse effects, ${ }^{37}$ and in this study, six participants (three patients and three controls, $20 \%$ of participants) were excluded as they did 
Table 1 Hippocampal volumes and subfield papers: 7T MRI in vivo studies including subjects with neurodegenerative dementia

\begin{tabular}{|c|c|c|c|c|}
\hline Paper & Imaging methods & Participants & Question & Results \\
\hline $\begin{array}{l}\text { Boutet } \\
\text { et } a^{67}\end{array}$ & $\begin{array}{l}\text { Whole brain: MP-RAGE acquisition; } 176 \text { slices; voxel } \\
\text { size } 0.9 \times 0.9 \times 0.9 \mathrm{~mm} \\
\text { Hippocampus: } 2 \mathrm{D} \mathrm{T} 2^{*} \text { GRE acquisition; } 3 \times 15 \text { slices; } \\
\text { voxel size } 0.3 \times 0.3 \times 1.2 \mathrm{~mm} \\
\text { Manual segmentation based on anatomical landmarks }\end{array}$ & $\begin{array}{l}\mathrm{NC}=7 \\
\text { 'mild to } \\
\text { moderate' } \\
\mathrm{AD}^{*}=4\end{array}$ & $\begin{array}{l}\text { Can volume changes in distinct } \\
\text { hippocampal layers be detected } \\
\text { in vivo using 7T MRI (using } \\
\text { imaging protocol distinguishing } \\
\text { between layers richer/poorer in } \\
\text { neuronal bodies)? }\end{array}$ & $\begin{array}{l}\text { Bilateral reduction of CA-SRLM and SUB-SP volumes } \\
\text { (sig) in AD (Average difference }-29 \% \text { to }-49 \% \text { ) } \\
\text { Trend towards reduction of left CA-SP in AD (non-sig) }\end{array}$ \\
\hline $\begin{array}{l}\text { Kerchner } \\
\text { et } a l^{\beta 5}\end{array}$ & $\begin{array}{l}\text { Whole brain: T1-weighted 3T MRI } \\
\text { MTL: } 2 \mathrm{D} \mathrm{T} 2^{*} \text {-weighted GRE acquisition; } 100 \text { slices } \\
\text { in } 15 \text { subjects and } 40 \text { slices in } 15 \text { subjects; slice gap } \\
0 \mathrm{~mm} \text { in } 15 \text { subjects and } 3 \mathrm{~mm} \text { in } 15 \text { subjects; voxel } \\
\text { size } 0.195 \times 0.195 \times 2 \mathrm{~mm} \text {; scan time } 9.6 \mathrm{~min} \\
\text { Manual segmentation based on anatomical landmarks }\end{array}$ & $\begin{array}{l}\mathrm{NC}=16 \\
\text { 'Mild' } \\
\mathrm{ADt}+14\end{array}$ & $\begin{array}{l}\text { Is tissue loss in hippocampal CA1 } \\
\text { apical neuropil layer seen on in } \\
\text { vivo MRI in mild AD? }\end{array}$ & $\begin{array}{l}\text { CA1 cell-body layer thickness and entire CA1 } \\
\text { subfield thickness not sig diff between groups } \\
\text { CA1-SRLM width decreased in AD (sig) } \\
\text { CA1 apical neuropil thickness decreased in AD (sig) } \\
\text { Left lateralised changes in AD } \\
\text { THV not sig diff between groups (measured at 3T) }\end{array}$ \\
\hline $\begin{array}{l}\text { Kerchner } \\
\text { et } a l^{25}\end{array}$ & $\begin{array}{l}\text { MTL: T2-weighted FSE acquisition; } 16-18 \text { slices; } \\
\text { voxel size } 0.166 \times 0.166 \times 1.5 \mathrm{~mm} \text { (interpolated } \\
\text { reconstruction from } 0.22 \times 0.22 \times 1.5 \mathrm{~mm} \text { acquired } \\
\text { voxels); scan time around } 10 \mathrm{~min} \\
\text { Manual and semiautomated segmentation based on } \\
\text { anatomical landmarks }\end{array}$ & Mild $A D \ddagger=9$ & $\begin{array}{l}\text { Do hippocampal subfield widths } \\
\text { correlate with episodic memory } \\
\text { task performance? }\end{array}$ & $\begin{array}{l}\text { Delayed recall performance (DRP) correlated with } \\
\text { CA-SRLM width } r^{2}=0.69 \text {, with CA1-SP width } r^{2}=0.5 \text {, } \\
\text { with ERC width } r^{2}=0.62 \text { (all sig) } \\
\text { Differences between groups left lateralised }\end{array}$ \\
\hline $\begin{array}{l}\text { Kerchner } \\
\text { et } a l^{26}\end{array}$ & As per Kerchner et a $\left.\right|^{25}$ & $\begin{array}{l}\mathrm{YNC}=9 \\
\mathrm{ONC}=18 \\
\mathrm{MCI}=15 \\
\mathrm{AD} \neq=11\end{array}$ & $\begin{array}{l}\text { Do ERC and CA1-SRLM } \\
\text { share early vulnerability to } \\
\text { AD pathology? (Is atrophy } \\
\text { proportional between the two } \\
\text { structures?) }\end{array}$ & $\begin{array}{l}\text { Ratio of CA1-SRLM to ERC width } 0.25 \pm 0.03 \text { across } \\
\text { all subjects } \\
\text { Width of CA1-SRLM and ERC decreased as function } \\
\text { of age and cognitive impairment } \\
\text { CA1-SRLM width and episodic memory performance } \\
\text { correlated in AD/ aMCl; not in controls } \\
\text { ERC width and episodic memory } \\
\text { performance correlated with AD (sig). Non-sig trend } \\
\text { in aMCl } \\
\text { ERC and CA1-SRLM widths correlated, and ERC and } \\
\text { CA1-SP widths correlated (sig when THVs controlled } \\
\text { for) } \\
\text { ERC and CA1-SRLM share vulnerability to age and } \\
\text { AD-associated atrophy }\end{array}$ \\
\hline $\begin{array}{l}\text { Kerchner } \\
\text { et } a l^{27}\end{array}$ & As per Kerchner et $a l^{25}$ & $\begin{array}{l}\mathrm{NC}=14 \\
\mathrm{MCI} \S=14 \\
\mathrm{AD}^{*}=11\end{array}$ & $\begin{array}{l}\text { Is there a relationship between } \\
\text { APOE e4, hippocampal subfield } \\
\text { morphology and episodic } \\
\text { memory? }\end{array}$ & $\begin{array}{l}\text { APOE e4 load associated with thinner CA1-SRLM } \\
\text { (sig) and poorer episodic memory task performance } \\
\text { (association not seen with other areas of cognitive } \\
\text { examination). Association persisted after controlling } \\
\text { for dementia severity } \\
\text { APOE e4 not associated with changes in other hippo } \\
\text { subfields or ERC }\end{array}$ \\
\hline $\begin{array}{l}\text { Wisse } \\
\text { et } a^{28}\end{array}$ & $\begin{array}{l}\text { MTL: 3D T2-weighted TSE (whole brain) acquisition; } \\
\text { voxel size } 0.35 \times 0.35 \times 0.7 \mathrm{~mm} \text {; scan time } 10: 15 \mathrm{~min} \\
\text { Manual in-house segmentation approach }\end{array}$ & $\begin{array}{l}N C=29 \\
M C l \|=16 \\
A D^{*}=9\end{array}$ & $\begin{array}{l}\text { Do hippo subfield and ERC } \\
\text { volumes differ between NC, } \\
M C l \text { and } A D \text { groups? Do hippo } \\
\text { subfield and ERC volumes } \\
\text { correlate with age in NCs? }\end{array}$ & $\begin{array}{l}\text { ERC, SUB, CA1, CA3, DG\&CA4, THV volumes smaller } \\
\text { in AD than NC (sig) } \\
\text { ERC, SUB, CA1, DG\&CA4, THV volumes smaller in AD } \\
\text { than MCI (sig) } \\
\text { MMSE correlated with ERC, SUB, CA1, DG\&CA4 } \\
\text { volumes (sig) } \\
\text { CA1, DG\&CA4, THV volume loss correlated with } \\
\text { increasing age (sig) }\end{array}$ \\
\hline $\begin{array}{l}\text { Wisse } \\
\text { et } a l^{43}\end{array}$ & $\begin{array}{l}\text { Hippocampal formation: } 7 \mathrm{~T} \text { 3D T2-weighted } \\
\text { TSE (whole brain) acquisition; voxel size } \\
0.35 \times 0.35 \times 0.7 \mathrm{~mm} \text {; scan time } 10: 15 \mathrm{~min} \\
\text { ICV: } 3 \mathrm{~T} \text { T2-weighted fast field echo; voxel size } \\
3 \times 0.99 \times 0.99 \mathrm{~mm} \text {; scan time } 2: 48 \text { min } \\
\text { Diffusion data: } 3 \mathrm{~T} \text { single-shot spin echo planar } \\
\text { imaging sequence (SENSE) (twice-refocused); voxel } \\
\text { size } 2.5 \times 2.5 \times 2.5 \mathrm{~mm} \text {; scan time } 5: 32 \mathrm{~min} \\
\text { Manual in-house segmentation approach }\end{array}$ & $\begin{array}{l}N C=17 \\
M C \mid q=15 \\
\text { mild } A D^{*}=10\end{array}$ & $\begin{array}{l}\text { Does ERC and hippocampal } \\
\text { degeneration induce } \\
\text { degeneration of associated } \\
\text { WM tracts (fornix and } \\
\text { parahippocampal cingulum)? }\end{array}$ & $\begin{array}{l}\text { Fornix FA lower in AD (sig) } \\
\text { CA1, DG\&CA4, SUB volumes lower in MCI/ AD (sig) } \\
\text { Fornix FA associated with SUB volume } \beta=0.53 \text { (sig) in } \\
\text { MCI/AD. Fornix FA not associated with ERC or other } \\
\text { hippo subfield volumes in MCI/AD } \\
\text { PHC FA not associated with ERC or other hippo } \\
\text { subfield volumes in MCI/AD } \\
\text { Hippo subfield atrophy not associated with reduction } \\
\text { in whole brain WM FA }\end{array}$ \\
\hline
\end{tabular}

AD diagnostic criteria used: $*^{2}$, tcriteria not specified, $\ddagger^{4}$.

MCl diagnostic criteria used: $\S^{23} q^{24}$

3D, three dimensional; $A D$, Alzheimer's disease; $B_{0^{\prime}}$ static field strength; $C[11]$-PiB, Carbon 11 Pittsburgh B compound (binds to amyloid); CA, cornu ammonis; $C S F$, cerebrospinal fluid; DG, dentate gyrus; EOAD, early-onset AD; ERC, entorhinal cortex; FA, fractional anisotropy; FSE/TSE, fast/turbo spin echo (these terms are synonymous); GM, grey matter; GRE, gradient echo sequence; hippo, hippocampus; ICV, intracranial volume; LOAD, late-onset AD; MCI, mild cognitive impairment; MMSE, Mini-Mental State Examination; MP- RAGE, magnetisation-prepared rapid acquisition gradient echo; MTL, medial temporal lobe; NC, cognitively normal controls; ONC, older cognitively normal controls; PHC, parahippocampal cingulum; QSM, quantitative susceptibility mapping; rs-fMRI, resting state functional MRI; RF, radio frequency; ROI, region of interest; sig, significant $(\mathrm{p}<0.05)$; SP, stratum pyramidale; SPACE, sampling perfection with application optimised contrasts by using different flip angle evolutions; SRLM, strata radiatum, lacunosum and moleculare; SUB, subiculum; SWI, susceptibility-weighted imaging; TE, echo time; THV, total hippocampal volume; TMP, temporoparietal region; WM, white matter; YOC, younger cognitively normal controls. 
Table 2 Microbleed, plaque and miscellaneous papers: 7T MRI in vivo studies including subjects with AD

\begin{tabular}{|c|c|c|c|c|}
\hline Paper & Imaging methods & Participants & Question & Results \\
\hline \multicolumn{5}{|c|}{ Microbleed and microinfarct papers: } \\
\hline Brundel et $\left.a\right|^{34}$ & $\begin{array}{l}\text { 7T MRI: dual-echo } 3 \mathrm{D} \mathrm{T2*} \text { - } \\
\text { weighted acquisition; voxel size } \\
0.5 \times 0.5 \times 0.7 \mathrm{~mm} \\
\text { 3T MRI: } 3 \mathrm{D} \mathrm{T2} 2^{*} \text {-weighted } \\
\text { acquisition; voxel size } \\
0.99 \times 0.99 \times 3 \mathrm{~mm} \\
\text { Visual identification of } \\
\text { microbleeds (MARS criteria) }\end{array}$ & $\begin{array}{l}\mathrm{NC}=18 \\
A D^{*} / \mathrm{MCl}+=18\end{array}$ & $\begin{array}{l}\text { What is the true prevalence } \\
\text { of microbleeds in } \mathrm{AD} / \mathrm{MCl} \text { ? }\end{array}$ & $\begin{array}{l}\text { At } 7 \mathrm{~T} \text { MRI } \geq 1 \text { microbleeds in } 78 \% \text { of } \mathrm{AD} / \mathrm{MCl} \text { vs } 44 \% \mathrm{NC} \\
\text { (sig) } \\
\text { At } 3 \mathrm{~T} \mathrm{MRI} \geq 1 \text { microbleeds in } 33 \% \text { of } \mathrm{AD} / \mathrm{MCl} \text { vs } 17 \% \mathrm{NC} \\
\text { (non-sig) } \\
\text { Higher quantity of microbleeds in AD/MCI (max } 80 \text { in one } \\
\text { subject) than NC (max } 5 \text { in one subject) at } 7 \mathrm{~T} \text { (sig) }\end{array}$ \\
\hline van Veluw et $a^{60}$ & $\begin{array}{l}\text { 3D FLAIR acquisition; voxel size } \\
0.8 \times 0.8 \times 0.8 \mathrm{~mm} \\
\text { 3D T2-weighted acquisition; voxel } \\
\text { size } 0.7 \times 0.7 \times 0.7 \\
\text { 3D T1-weighted acquisition; voxel } \\
\text { size } 1.0 \times 1.0 \times 1.0 \mathrm{~mm} \\
\text { 3D dual-echo gradient } \\
\text { echo acquisition; voxel size } \\
0.5 \times 0.5 \times 0.7 \mathrm{~mm} \\
\text { Semiautomated identification of } \\
\text { microbleeds }^{69}\end{array}$ & $\begin{array}{l}\mathrm{NC}=22 \\
\mathrm{AD} / \mathrm{aMCl}+=29\end{array}$ & $\begin{array}{l}\text { Are cerebral microinfarcts } \\
(\mathrm{CMI}) \text { increased in } \mathrm{aMCl} / \\
\mathrm{AD} \text { ? }\end{array}$ & $\begin{array}{l}\text { CMIs found in } 45 \% \text { NC and } 55 \% \text { aMCI/AD (non-sig) } \\
\text { No sig diff between CMls in aMCI and AD } \\
\text { In all subjects, CMIs uniformly distributed throughout } \\
\text { cortex } \\
\text { No relationship between MTL atrophy or MMSE and } \\
\text { number of CMls }\end{array}$ \\
\hline \multicolumn{5}{|l|}{ Amyloid plaque papers: } \\
\hline Nakada et al $(2008)^{30}$ & $\begin{array}{l}\text { Parietal association cortex SWI: } \\
\text { T2*-weighted 2D GRE acquisition; } \\
\text { voxel size } 0.156 \times 0.156 \times 3 \mathrm{~mm} ; \\
\text { scan time } 3: 48 \mathrm{~min} \\
\text { Visual identification of plaques } \\
\text { described in Ref. } 70\end{array}$ & $\begin{array}{l}\mathrm{ONC}=10 \\
\mathrm{YNC}=20 \\
A D^{*}=10\end{array}$ & $\begin{array}{l}\text { Can senile plaques be } \\
\text { visualised using } 7 T \text { MRI in } \\
\text { vivo in } A D \text { ? }\end{array}$ & $\begin{array}{l}\text { Hypointense 'black dots' ('senile-plaque-like- } \\
\text { pathology') seen throughout parietal cortex in 10/10 AD, in } \\
2 / 10 \text { ONC, in } 0 / 20 \text { YNC } \\
\text { Sig diff between YNC and ONC and between AD and ONC } \\
\text { (Ryan multiple comparison) }\end{array}$ \\
\hline van Bergen et $a l^{\beta 3}$ & $\begin{array}{l}\text { Structural: T1-weighted MP2- } \\
\text { RAGE acquisition; voxel size } \\
0.6 \times 0.6 \times 0.6 \mathrm{~mm} \text {; scan time } \\
\text { 7:50 min } \\
\text { QSM: } 3 \text { D GRE acquisition } \\
\text { with } 3 \text { echoes; voxel size } \\
0.5 \times 0.5 \times 0.5 \mathrm{~mm} \text {; scan time } \\
\text { 13:48 min } \\
\text { rs-fMRI: } 3 \text { D T2-prep GRE } \\
\text { acquisition; voxel size } \\
\text { 1.5×1.5×1.5 mm; scan time } \\
\text { 7:03 min } \\
\text { Also: C[11]-PiB-PET } \\
\text { QSM maps created in multiple } \\
\text { steps. Laplacian-based phase } \\
\text { unwrapping converted to } \\
\text { frequency shift images (in hertz), } \\
\text { inverse dipole calculation used to } \\
\text { obtain susceptibility maps, values } \\
\text { reported relative to reference } \\
\text { CSF }{ }^{71} \\
\text { rs-fMRI images created using } \\
\text { SPM12 (http://www.fil.ion.ucl. } \\
\text { ac.uk/ } \\
\text { spm/) }\end{array}$ & $\begin{array}{l}\mathrm{NC}=22 \text { (7APOE e4 } \\
\text { positive) } \\
\text { MCl }=15 \text { (6APOE } \\
\text { e4 positive) }\end{array}$ & $\begin{array}{l}\text { What is the relationship } \\
\text { between cerebral iron (using } \\
\text { QSM) MCI and APOE-e4 } \\
\text { status? } \\
\text { What are the relationships } \\
\text { between cerebral iron } \\
\text { burden, Aß -plaque density } \\
\text { and MPFC-coupling (using } \\
\text { rs-fMRI)? }\end{array}$ & $\begin{array}{l}\text { APOE e4 associated with increased cortical iron and higher } \\
\text { A } \beta \text {-plaque-load in } \mathrm{MCI}(\mathrm{sig}) \text {; not in NC } \\
\text { High iron burden in } \mathrm{MCl} \text { associated with increased MPFC- } \\
\text { coupling in ROIs, including cingulate, paracingulate, frontal } \\
\text { regions (sig) } \\
\text { In areas with increased MPFC coupling iron-burden and } \\
\text { A } \beta \text {-plaque-load correlated (sig) }\end{array}$ \\
\hline van Rooden et $a \beta^{31}$ & $\begin{array}{l}\text { T2*-weighted 2D GRE } \\
\text { acquisition; } 20 \text { slices; voxel size } \\
0.24 \times 0.24 \times 1 \mathrm{~mm} \text { (FOV included } \\
\text { frontal and parietal regions); } \\
\text { scan time approximately } 10 \mathrm{~min} \\
\text { Hippocampus: } 2 \mathrm{D} \text { T2*-weighted } \\
\text { GRE acquisition; } 32 \text { slices; voxel } \\
\text { size } 0.5 \times 0.5 \times 3 \mathrm{~mm} \text {; scan time } \\
\text { approximately } 6 \mathrm{~min} \\
\text { Visual identification of plaques } \\
\text { Phase shift values for four } \\
\text { different ROls (right and left } \\
\text { TMP, frontal and parietal) } \\
\text { averaged and phase shift with } \\
\text { subcortical WM calculated }\end{array}$ & $\begin{array}{l}N C=15 \\
A D^{*}=16\end{array}$ & $\begin{array}{l}\text { Can amyloid plaques be } \\
\text { visualised in the cerebral } \\
\text { cortex using } 7 T \text { MRI in vivo } \\
\text { in } A D ?\end{array}$ & $\begin{array}{l}\text { No focal hypointensities found } \\
\text { Increased cortical phase shift in left } T M P(A D=0.90 \text {, } \\
N C=79 \text { ), right } T M P(A D=0.97, N C=0.85) \text {, frontal region } \\
(A D=0.70, N C=0.62) \text {, parietal region }(A D=0.87, N C=0.74) \\
\text { in } A D \text { (all sig) } \\
\text { Phase shift difference between groups in hippo (left } \\
\text { hippo } A D=0.09, N C=0.07 \text {; right hippo } A D=0.10 \text {, } \\
N C=0.08 \text { ) not sig diff } \\
\text { Association between whole brain phase shift and MMSE } \\
\text { scores } r=-0.54 \text { (sig) }\end{array}$ \\
\hline
\end{tabular}


Table 2 Continued

\begin{tabular}{|c|c|c|c|c|}
\hline Paper & Imaging methods & Participants & Question & Results \\
\hline van Rooden et $a l^{\beta 2}$ & $\begin{array}{l}\text { T2*-weighted 2D GRE } \\
\text { acquisition; } 20 \text { slices; voxel size } \\
0.24 \times 0.24 \times 1 \mathrm{~mm} \text {; scan time } \\
\text { approximately } 10 \mathrm{~min} \text { (FOV } \\
\text { included frontal and parietal } \\
\text { regions) } \\
\text { Phase shift values for four } \\
\text { different ROls (right and left TMP, } \\
\text { frontal and parietal) averaged and } \\
\text { phase shift with subcortical WM }\end{array}$ & $\begin{array}{l}\mathrm{NC}=27 \\
E O A D^{*}=12 \text { (onset } \\
\text { before } 65 \text { years) } \\
\mathrm{LOAD}^{*}=17 \text { (onset } \\
\text { after } 65 \text { years) }\end{array}$ & $\begin{array}{l}\text { Does amyloid deposition } \\
\text { (measured as per van } \\
\text { Rooden et } a l)^{31} \text { differ } \\
\text { between EOAD and LOAD? }\end{array}$ & $\begin{array}{l}\text { Increased cortical phase shift in } \angle O A D / E O A D \text { vs } N C \text { in } \\
\text { right } T M P(L O A D=1.23, E O A D=1.31, N C=0.98) \text {, left } T M P \\
(L O A D=1.18, E O A D=1.25, N C=0.96) \text {, frontal }(\angle O A D=1.04 \text {, } \\
E O A D=1.15, N C=0.88) \text {, parietal }(\angle O A D=1.16, E O A D=1.27 \text {, } \\
N C=0.98) \text { regions and whole brain }(L O A D=1.15 \text {, } \\
E O A D=1.25, N C=0.95) \\
\text { Phase shift in all regions and whole brain sig diff between } \\
N C \text { and } L O A D \text {, between } N C \text { and } E O A D \text {, between } L O A D \text { and } \\
E O A D \text { groups }\end{array}$ \\
\hline
\end{tabular}

Miscellaneous papers:

\begin{abstract}
Cai et $a l^{72}$
\end{abstract}
Versluis et a ${ }^{44}$

phase shift with subcortical WM

calculated

\begin{tabular}{|c|c|}
\hline & $\begin{array}{l}\text { acquisition; } 224 \text { slices; voxel } \\
\text { size } 0.4 \times 0.4 \times 0.4 \mathrm{~mm} \text {; scan } \\
\text { time approximately } 7.5 \mathrm{~min} \\
\text { (interpolated reconstruction from } \\
0.42 \times 0.42 \times 1 \mathrm{~mm} \text { acquired voxels) } \\
\text { PVS automatically segmented } \\
\text { using MATLAB }\end{array}$ \\
\hline Versluis et $a l^{44}$ & $\begin{array}{l}\text { T2* -weighted acquisition with } \\
\text { additional navigator echo } \\
\text { technique application; } 20 \text { slices; } \\
\text { voxel size } 0.24 \times 0.24 \times 1 \mathrm{~mm} \text {; scan } \\
\text { time approximately } 10 \mathrm{~min}\end{array}$ \\
\hline
\end{tabular}

\section{$\mathrm{NC}=3$ \\ $A D \ddagger=5$ \\ Increase in PVS density in AD vs NC (AD=8.0, NC=4.9) (sig) \\ Can PVS be imaged and}

AD +5

(2)
in vivo?

AD diagnostic criteria used: * ${ }^{2}$

$\mathrm{MCl}$ diagnostic criteria used: $t^{24}$

$A D$ diagnostic criteria used: $¥ c r i t e r i a$ not specified.

3D, three dimensional; $A \beta$, amyloid beta; $A D$, Alzheimer's disease; $B 0$, static field strength; $C[11]$-PiB, Carbon 11 Pittsburgh B compound (binds to amyloid); $C A$, cornu ammonis; CSF, cerebrospinal fluid; DG, dentate gyrus; EOAD, early-onset AD; ERC, entorhinal cortex; FA, fractional anisotropy; FSE/TSE, fast/turbo spin Echo (these terms are synonymous); GM, grey matter; GRE, gradient echo sequence; hippo, hippocampus; ICV, intracranial volume; LOAD, late-onset AD; MCl, mild cognitive impairment; MMSE, Mini-Mental State Examination; MP2-RAGE, magnetisation-prepared 2 rapid acquisition gradient echo; MTL, medial temporal lobe; NC, cognitively normal controls; ONC, older cognitively normal controls; PHC, parahippocampal cingulum; PVS, perivascular space; QSM, quantitative susceptibility mapping; rs-fMRI, resting state functional MRI; RF, radio frequency; ROI, region of interest; sig, significant ( $\mathrm{p}<0.05)$; SP, stratum pyramidale; SPACE, sampling perfection with application optimised contrasts by using different flip angle evolutions; SRLM, strata radiatum, lacunosum and moleculare; SUB, subiculum; SWI, susceptibility-weighted imaging; TE, echo time; THV, total hippocampal volume; TMP, temporoparietal region; WM, white matter; YOC, younger cognitively normal controls.

not tolerate 7T MRI; however, the reasons behind this were not elaborated on.

\section{DISCUSSION}

\section{Hippocampal subfield papers investigating $A D$ and $\mathrm{MCI}$}

The hippocampus is not a uniform structure-it consists of multiple subfields (CA-1, 2, 3 and 4, subiculum and dentate gyrus. ${ }^{40}$ Postmortem studies suggest early involvement of ERC, subiculum, CA1 and dentate gyrus in $\mathrm{AD},{ }^{41}$ and hippocampal atrophy is well established as a biomarker for AD. ${ }^{42}$ It is possible to delineate the subfields of the hippocampus using 3T MRI in vivo; at $1.5 \mathrm{~T}$ and $3 \mathrm{~T}$ CA1, CA2 and subiculum atrophy (using both field strengths to image the same participants) was found in AD but not in MCI. ${ }^{17}$ In this paper, improved SNR and greater effect sizes were noted at $3 \mathrm{~T}$ (compared with $1.5 \mathrm{~T}$ ), suggesting that a similar further improvement might be seen with an increase of $\mathrm{B}_{0}$ to $7 \mathrm{~T}$. Papers reviewed here found significant atrophic changes in MCI compared with controls, ${ }^{26} 2843$ suggesting that improved resolution, SNR and consequent increased effect size has real benefits, allowing differentiation of MCI and AD from controls at 7T. Changes in CA1-SRLM width in mild AD in the absence of differences in total CA1 or total hippocampal widths were also reported, possibly demonstrating very early changes, previously undetected on MRI. ${ }^{35}$ The average between group difference in CA1-SRLM in this paper was around 1 voxel, therefore, at the limit of detectable changes, further emphasising the advantages of $7 \mathrm{~T}$ MRI. MCI is of particular interest in AD research, accurate identification of which in this group develop $\mathrm{AD}$ may allow earlier diagnosis and allow the targeted treatment of 'prodromal AD' before substantial memory and functional deficits are apparent.

As suggested by postmortem findings, the papers reviewed here consistently report reductions in CA1 (specifically CA1-SRLM); however, there was less consensus regarding changes in other subfields. For example, Kerchner et al found that hippocampal volume reductions were restricted to CA1-SRLM, ${ }^{35}$ while Wisse et al noted volume reductions throughout the majority of subfields. ${ }^{28}$ Imaging protocols were reported to be similar in these studies, and differences in segmentation approaches, different degrees of dementia and small numbers of subjects may account for the lack of consensus. There is consensus in the papers reviewed that 7T MRI produces images of sufficiently high resolution to allow improved visualisation of hippocampal substructures in vivo. However, while high in-plane resolution allowed visualisation of the fine detail of the hippocampal formation, due to the trade-off between high in-plane resolution and slice thickness, it was not possible to use differences in microstructure to segment the hippocampal formation throughout the length of the hippocampus in vivo. Protocols described in these papers resorted to macrostructure rather than 


\begin{tabular}{|c|c|c|c|c|}
\hline Paper & Imaging methods & Participants & Question & Results \\
\hline Apple et $a l^{37}$ & $\begin{array}{l}\text { Volumetric: inversion recovery T1-weighted } \\
\text { acquisition; voxel size } 1 \times 1 \times 1 \mathrm{~mm} \text {; scan time } \\
3: 33 \mathrm{~min} \\
\text { Phase imaging: GRE acquisition; voxel size } \\
0.5 \times 0.5 \times 4 \mathrm{~mm} \\
\text { Also } 3 \mathrm{~T} \text { volumetric T1-weighted acquisition; scan } \\
\text { time 6:18 min } \\
\text { Phase images constructed with fully automated } \\
\text { phase unwrapping algorithm }\end{array}$ & $\begin{array}{l}\mathrm{NC}=13 \\
\text { Pre-HD* }=13 \\
\text { (originally } 16 \\
\text { per group, with } \\
6 \text { excluded due } \\
\text { to not tolerating } \\
\text { MRI) }\end{array}$ & $\begin{array}{l}\text { Do quantitative 7TMR } \\
\text { phase measurements of } \\
\text { 7T LFS within CN differ } \\
\text { between groups? }\end{array}$ & $\begin{array}{l}\text { Higher CN LFS in premotor HD vs NC (sig) } \\
\text { Strong correlation between LFS and CAP } \\
\text { score }\left(R^{2}=0.61 \text {, sig) }\right.\end{array}$ \\
\hline van Bergen et a ${ }^{\beta 8}$ & $\begin{array}{l}\text { T1-weighted MP-RAGE acquisition; voxel size } \\
0.6 \times 0.6 \times 0.6 \mathrm{~mm} \text {; scan time 6:32 min } \\
\text { QSM: multiecho 3D GRE acquisition; voxel size } \\
\text { 1×1×1 mm; scan time 6:12 min } \\
\text { QSM maps created in multiple steps; Laplacian- } \\
\text { based phase unwrapping converted to frequency } \\
\text { shift images (in hertz), inverse dipole calculation } \\
\text { used to obtain susceptibility maps, values reported } \\
\text { relative to reference region (CSF in lateral } \\
\text { ventricles) } \\
\text { R2* maps calculated using the power method }\end{array}$ & $\begin{array}{l}\mathrm{NC}=16 \\
\text { Pre-HDt=15 }\end{array}$ & $\begin{array}{l}\text { Do groups show } \\
\text { differences in magnetic } \\
\text { susceptibility (proxy } \\
\text { measure of iron } \\
\text { deposition) in basal } \\
\text { ganglia structures in vivo } \\
\text { using QSM and R2* at 7T } \\
\text { MRI? }\end{array}$ & $\begin{array}{l}\text { Increased magnetic susceptibility in } \mathrm{CN} \text { and } \\
\text { putamen }(\sim 2 x(\text { sig)) in pre-HD (correlation } \\
\text { with CAP score and volume reductions) } \\
\text { Increased magnetic susceptibility in GB (sig) } \\
\text { in pre-HD } \\
\text { Decreased magnetic susceptibility in SN and } \\
\text { hippocampus in pre-HD (sig) } \\
\text { Increased R2* values in CN and putamen in } \\
\text { pre-HD (sig); R2*findings only in these two } \\
\text { regions and effect size smaller }\end{array}$ \\
\hline Doan et $a l^{75}$ & $\begin{array}{l}\text { 3D T1-weighted GRE acquisition; voxel size } \\
0.3 \times 0.3 \times 2 \mathrm{~mm} \\
3 \mathrm{D} \mathrm{T} 2^{*} \text {-weighted GRE acquisition; voxel size } \\
0.25 \times 0.25 \times 0.5 \mathrm{~mm} \\
\text { Also } 3 \mathrm{~T} \text { T1-weighted volumetric acquisition; } 164 \\
\text { slices; voxel size } 1.1 \times 1.0 \times 1.0 \mathrm{~mm} \\
\text { Textural features analysed in ROls using a 3D } \\
\text { Grey-Level Co-occurrence Matrix }{ }^{76}\end{array}$ & $\begin{array}{l}\mathrm{NC}=5 \\
\mathrm{HD}=8 \ddagger \\
\text { Pre- } \mathrm{HD} \S=7\end{array}$ & $\begin{array}{l}\text { Are there textural } \\
\text { differences in subcortical } \\
\text { structures between } \\
\text { groups? }\end{array}$ & $\begin{array}{l}\text { No sig diff between textural features in } \\
\text { subcortical structures in pre-HD vs NC when } \\
\text { Bonferroni applied (without correction } \\
\text { sig diff in CN) } \\
\text { Sig diff between textural features in } \\
\text { putamen in HD vs NC when Bonferroni } \\
\text { applied (without correction sig diff in } \\
\text { pallidum, CN, putamen,thalamus) }\end{array}$ \\
\hline $\begin{array}{l}\text { Drouin-Ouellet et al }{ }^{77} \\
\text { NB/Expansive study } \\
\text { using mouse model, } \\
\text { 3T and 7T MRI and } \\
\text { postmortem tissue } \\
\text { analysis. 7T MRI part of } \\
\text { paper examined here }\end{array}$ & $\begin{array}{l}\text { LL-EPI F-SAIR ASL acquisition protocol; voxel size } \\
2 \times 2 \times 4 \mathrm{~mm} \\
\text { PSIR anatomical acquisition; voxel size } \\
0.8 \times 0.8 \times 0.8 \mathrm{~mm} \\
\text { Turbo field EPI (MR angiogram); } 100 \text { slices; } \\
0.6 \times 0.6 \times 0.6 \mathrm{~mm} \\
\text { Average aCBV (mL blood } / 100 \mathrm{~mL} \text { tissue) } \\
\text { and arterial transit time calculated using } 2 \\
\text { compartment vascular kinetic model }^{78}\end{array}$ & $\begin{array}{l}\text { In } 7 \mathrm{~T} \text { part: } \\
\mathrm{NC}=7 \\
\mathrm{HD} \|=8\end{array}$ & $\begin{array}{l}\text { Are differences in aCBV } \\
\text { (representing changes in } \\
\text { cerebral vasculature) seen } \\
\text { between groups? }\end{array}$ & $\begin{array}{l}\text { Increase in cortical GM aCBV in HD (sig). } \\
\text { No correlation between aCBV and GM } \\
\text { volume } \\
\text { No difference in aCBV in caudate or putamen }\end{array}$ \\
\hline
\end{tabular}

\footnotetext{
HD diagnostic criteria used: positive for HD mutation with $\geq 40$ repeats.

${ }^{*}$ UHDRS motor subscale score $\leq 3$. † UHDRS motor subscale score $\leq 15$.

fUHDRS motor subscale score $\geq 5$.

$\S U H D R S$ motor subscale score $\leq 5$.

INo UHDRS motor score cut-off; pre-HD diagnostic criteria used: positive for HD mutation with $\geq 40$ repeats.

3D, three dimensional; aCBV, arterial cerebral blood volume; CAP score, CAG-Age Product Scaled score; CN, caudate nucleus; CSF, cerebrospinal fluid; EPI, echo planar imaging; GM, grey matter; GP, globus pallidus; GRE, gradient echo sequence; HD, Huntington's disease; LFS, local field shift (a measure of phase and proxy measure of iron deposition); LL-EPI F-SAIR ASL, Look-Locker echo planar imaging flow-sensitive alternating inversion recovery arterial spin labelling (used to generate arterial cerebral blood volume maps); MP2-RAGE, magnetisation-prepared 2 rapid acquisition gradient echo; NC, cognitively normal controls; pre-HD, premanifest HD; PSIR, phase-sensitive inversion recovery; R2*, apparent spin-spin relaxation rate; sig, significant $(\mathrm{p}<0.05)$; SN, substantia nigra; UHDRS, Unified Huntington's Disease Rating Scale.
}

microstructure to differentiate between some of the subfield layers $\left(\mathrm{eg},{ }^{31}\right)$.

Most of the studies reviewed described their participants as 'mild' or 'early' AD, and many stipulated that all participants have a minimum MMSE of 20. This is likely due to the practicalities of imaging subjects with more advanced AD (issues include consent, compliance and excessive within-scanner movement) and may mean that results are not generalisable to the wider patient population. Versluis et al suggested that the challenge of increased artefact production due to movement can be largely overcome by modifications of image acquisition sequence and in postprocessing. ${ }^{44} \mathrm{~A}$ navigator echo was used, in which physiological movement such as that caused by respiration and pulse is tracked using additional RF pulses, and a correction for this is factored in when the MRI signal is converted into k-space. Excessive motion artefact can also be corrected prospectively, for example, using an optical tracking system where a marker is attached to a dental plate, which is held in the subject's mouth. ${ }^{45}$
However, such methods require an extremely cooperative participant and may be uncomfortable, and for patient participants in dementia studies, such methods may not be ideal.

None of the reviewed papers reported serial MRI changes at 7T, which is an established biomarker for clinical trials. ${ }^{46} \mathrm{~A}$ lack of longitudinal data limits our understanding of how the changes seen relate to the natural history of these diseases. For example, Wisse et al investigated whether ERC and hippocampal degeneration induced degeneration of associated WM tracts ${ }^{43}$; however, investigating the likely order of events within a disease process without using longitudinal data means that conclusions are difficult to reach, and in this paper, it is possible that WM tracts degenerate first, causing associated cortical tissue regions to atrophy in a proportional manner.

Hippocampal subfield pathology is also reported in Parkinson's disease, PDD and LBD. On postmortem, hippocampal pathology is seen primarily in CA2, CA3 and dentate gyrus rather than $\mathrm{CA} 1$, which is primarily affected in $\mathrm{AD} .{ }^{47}$ Findings 
from in vivo MRI studies are conflicting. At 1.5T MRI, atrophy has been reported in CA1 and subiculum in LBD compared with controls. ${ }^{48}$ At $3 \mathrm{~T}$ MRI, CA1 has been found to be relatively preserved in LBD compared with $\mathrm{AD}$ and not to differ significantly from controls, while significant reductions have been found in whole hippocampal volumes and in CA2-3 in both LBD and $\mathrm{AD}$ compared with controls. ${ }^{49}$ The differentiation between hippocampal subfields on MRI has proved difficult at $1.5 \mathrm{~T}$ and $3 \mathrm{~T}^{50}$ which may account for differing results between studies. Seven tesla MRI, which begins to approach the scale required to illuminate the histological changes seen in postmortem studies, may be beneficial in confirming to what extent hippocampal subfield volume measurements differ between $\mathrm{AD}, \mathrm{LBD}$ and controls. In this review, we did not identify any published studies examining hippocampal subfields in LBD or FTD using 7T MRI.

\section{Amyloid plaque studies investigating $A D$ and $\mathrm{MCl}$}

Amyloid plaques are a cardinal feature of $\mathrm{AD}$, seen initially in the basal neocortex and later widely distributed throughout the cortex. ${ }^{3}$ PET has been highly successful in imaging neural amyloid deposition using amyloid-binding ligands such as $11[\mathrm{C}]$ PiB. ${ }^{51}$ One major advantage of developing a method of imaging amyloid using in vivo MRI lies in its much higher spatial resolution; specific identification of where in the brain amyloid is deposited at each stage of the disease process may be possible.

Amyloid plaques have high iron content, produce free-radicals and include iron-containing activated microglia. The $7 \mathrm{~T}$ in vivo plaque studies in this review exploited the increased sensitivity to susceptibility effect seen in brain tissues with abnormal quantities of amyloid. These papers concluded that the most likely origin of directly visualised hypointense areas and increased regional phase shift was amyloid, and this was supported somewhat by the only paper identified in which C[11]-PiB-PET was performed alongside susceptibility-weighted MRI. ${ }^{33}$ In the study of van Bergen et al, in APOE e4-positive individuals with MCI, brain regions with high susceptibility sensitivity (considered to reflect neural iron load) on MRI had also increased amyloid on PET. However, a more general correlation between susceptibility effects and amyloid across groups was not reported. An association was found between amyloid deposition and APOE e4 status but not between iron and MCI, independent of APOE e4 status. This may suggest that iron and amyloid are independently involved in the disease process. Postmortem studies correlating susceptibility-weighted 7T MRI with histological staining for iron in the same samples report inconsistent findings, and correlation between MRI hypointensities is reported with both amyloid deposits ${ }^{52}$ and with microscopic iron and activated microglia. ${ }^{53}$ Consequently, explanations other than (or in addition to) amyloid should also be considered. Susceptibility effects are also produced by iron, haemosiderin, deoxyhaemoglobin, methaemoglobin, free radicals and free oxygen. ${ }^{30}$ It may be that changes in these substrates produced the effects seen independently of amyloid burden and may be implicated in the $\mathrm{AD}$ disease process. Findings from histological studies suggest that changes in iron deposition and distribution may also play a key role in $\mathrm{AD}$ pathology. ${ }^{54}$ Disorders of iron homeostasis have also been postulated in PD and related disorders ${ }^{55}$ and FTD. ${ }^{56}$

Microbleed and microinfarct papers investigating AD and $\mathrm{MCI}$ Advantages of $7 \mathrm{~T}$ over $1.5 \mathrm{~T}$ MRI in the direct visualisation of microbleeds has been demonstrated. Microbleeds were detected in significantly more patients with atherosclerotic disease using 7T MRI (50\% of subjects) than using $1.5 \mathrm{~T}$ ( $21 \%$ of subjects). ${ }^{5}$
A review of $1.5 \mathrm{~T}$ MRI studies suggested that microbleed prevalence in $\mathrm{AD}$ is around 23\%. ${ }^{58}$ Imaging at both $3 \mathrm{~T}$ and $7 \mathrm{~T}$ in the same subjects, Brundel et al reported a microbleed prevalence of $33 \%$ in $\mathrm{AD}$ at $3 \mathrm{~T}$ (difference from controls was not significant) and of $78 \%$ at $7 \mathrm{~T}$ (with a significant difference between $\mathrm{AD}$ and control groups). ${ }^{34}$ The increase in in-plane resolution was from $0.99 \times 0.99 \mathrm{~mm}$ at $3 \mathrm{~T}$ to $0.5 \times 0.5 \mathrm{~mm}$ at $7 \mathrm{~T}$. This paper suggests that increasing $\mathrm{B}_{0}$ to $7 \mathrm{~T}$ allows the visualisation of pathology not previously evident via MRI.

Histopathology and ex vivo 7T MRI studies suggest an increased prevalence of microinfarcts in $\mathrm{AD}$ brains compared with those of controls. ${ }^{59}$ No such significant difference was found between $\mathrm{AD}$ and controls using 7T MRI in vivo, ${ }^{60}$ which may be accounted for by differences in subject characteristics. Participants in the study of van Veluw et $a l^{60}$ had a minimum MMSE of 20/30, while postmortem subjects are likely to have died with more advanced AD. Including subjects with more advanced disease or longitudinal analysis is required to illuminate the true prevalence of microinfarcts in $\mathrm{AD}$ and how they contribute to the clinical presentation. There are implications for our understanding of $\mathrm{AD}$ pathology and for $\mathrm{AD}$ diagnosis if 7T 'reveals' changes not previously visible. For example, the current diagnostic criteria preclude the diagnosis of $\mathrm{AD}$ in the presence of extensive infarcts or microbleeds, ${ }^{4}$ and at present, individuals with microbleeds or microinfarcts may be excluded from AD studies. ${ }^{3458}$

\section{Ultra-high-resolution MRI papers investigating HD and premanifest HD}

The 7T in vivo HD studies investigating iron deposition in premanifest $\mathrm{HD}^{34} 37$ used techniques similar to those previously described for imaging amyloid and microbleeds in AD and MCI. Findings suggesting increased neural iron in basal ganglia structures, including the caudate nucleus, mirror those found at lower field strengths and at postmortem. ${ }^{61}$ While using susceptibility effects as a proxy for iron burden corresponds well to prior postmortem findings, susceptibility effects in MRI may be caused by a number of different substrates, as previously described. Calculating apparent spin-spin relaxation rate $(\mathrm{R} 2 *)$ has been validated as a quantitative measure of iron in brain tissues. ${ }^{62}$ As $\mathrm{R} 2 *$ signal increases with $\mathrm{B}_{0}$, this is a further potential benefit of $7 \mathrm{~T}$ imaging. van Bergen et al reported increased $\mathrm{R} 2 *$ values in the caudate and putamen in premanifest HD but not in other basal ganglia structures, and effect sizes were smaller than those seen when using QSM. ${ }^{38}$ This might indicate that iron is only one contributor to the susceptibility effects seen in the basal ganglia in this study, and the authors suggest demyelination may also contribute. Many of the papers included in this review have benefitted from the increased sensitivity to susceptibility seen at 7T and have used this effect to variously measure amyloid, microbleeds and iron deposition. That the same or similar techniques, including QSM, SWI, R2* and phase shift, have been used to measure different processes is problematic, implying a lack of consensus regarding which pathological processes are actually producing changes in these measures on MRI. Ongoing research comparing histological findings, postmortem MRI and polymodal imaging (eg, comparing amyloid PET with ultra-high-resolution MRI) may provide future clarification.

A major limitation of HD papers in this review is the variable definition of premanifest HD versus HD. Each HD paper used different UHDRS motor score cut-off values. A lack of consistency within the literature complicates between-paper 
comparison and makes it difficult to correlate MRI biomarkers with clinical findings.

\section{Ultra-high-resolution imaging in dementias other than $A D$ and HD}

No in vivo studies using 7T MRI in neurodegenerative dementias other than AD or HD were identified in this review. Proportionally, more dementia cases are identified as LBD on postmortem than are diagnosed clinically, ${ }^{63}$ suggesting that LBD is under-recognised and may benefit from the identification of biomarkers to facilitate diagnosis in life. A lack of 7T MRI LBD studies may reflect the novelty of $7 \mathrm{~T} \mathrm{MRI,} \mathrm{the} \mathrm{dominance} \mathrm{of} \mathrm{AD}$ research, the relatively few individuals diagnosed with LBD compared with $\mathrm{AD}$ or the specific difficulties of imaging in this patient group, in which fluctuating alertness and movement disorders are core clinical features.

Postmortem and imaging studies have identified changes in small, complex brain structures in LBD, for example, in hippocampal subfields, substantia innominate, putamen, pons and thalamus. ${ }^{1048}$ Similar to the hippocampus, the thalamus is extremely complex structurally, and success has been reported in differentiating its substructure using 7T MRI in cognitively normal subjects. ${ }^{64}$ The AD studies in this review suggest that the challenges of imaging small, peripheral brain areas can be overcome with specialised acquisition sequences. Increased ghosting, artefacts and image drop-out do not appear to be as problematic as predicted. This suggests that areas of interest in LBD, such as the thalamus and basal ganglia, may benefit from $7 \mathrm{~T}$ imaging in a similar way.

No FTD studies were identified in this review. Although less common than AD or LBD, FTD is particularly important clinically due to its over-representation in younger onset dementias. ${ }^{65}$ The heterogeneous nature of FTD both clinically and pathologically and the relatively small numbers of individuals affected by this type of dementia may contribute towards this deficit. Distinct atrophic changes in the frontal and anterior temporal lobes is described for all subtypes at 1.5 and $3 \mathrm{~T}^{66}$ As is the case for $\mathrm{AD}$ and $\mathrm{LBD}$, imaging at ultra-high resolutions may be beneficial in describing the disease processes more fully in vivo and in developing biomarkers that can be used to monitor disease progression, guide prognosis, and be used to evaluate the effects of any potential treatments.

\section{CONCLUSIONS}

This systematic review identified 19 papers using 7T MRI in vivo to investigate neurodegenerative dementias. Fifteen of the papers identified were concerned with $\mathrm{AD}$, and the majority investigated hippocampal subfields. Four of the papers identified were concerned with HD and/or premanifest HD. The papers, in general, have four main limitations. First, participant numbers are small, which limits the power of these studies. Second, studies to date have been limited to $\mathrm{AD} / \mathrm{MCI}$ and $\mathrm{HD} /$ premanifest HD. Third, none have taken a longitudinal approach. Finally, few of the studies related imaging findings to detailed neuropsycholog$\mathrm{ical} /$ cognitive testing. A great advantage of in vivo over postmortem studies is the ability to link imaging findings to the clinical syndromes experienced by patients. The relevance of studies that do not explore the relationship between imaging and clinical findings may be limited. It is likely that as 7T MRI becomes more widely available, studies will include larger samples and produce longitudinal data, allowing researchers to examine the changes seen as dementias develop and illuminating prognoses, for example, by identifying which individuals with MCI will go on to develop AD.

In the small number of papers as yet published, the predicted challenges of ultrahigh resolution, such as increased artefact production, ghosting and image drop-out, are not much commented on in the papers. This implies that practical difficulties are less troublesome than the theory would suggest, and new technologies can overcome at least some of the issues. Total scan times were reported in 14 of the 19 papers reviewed and varied greatly according to the acquisition sequence used, FOV and voxel size. Increasing $\mathrm{B}_{0}$ allows faster acquisition times or increased resolution. In these papers, authors have not consistently sought to draw on one or the other of these potential benefits. We may conclude that consensus on ideal imaging parameters at $7 \mathrm{~T}$ in the dementia population has not yet been reached.

Of the papers reviewed, only three reported on scan tolerability or adverse effects for subjects, despite the theoretical increased risks at $7 \mathrm{~T}$ compared with lower field strengths. The low attrition rate in the studies and the lack of reported side effects may lead us to conclude that adverse effects were not often experienced by subjects in these studies. However, 7T MRI in vivo remains relatively new, and the populations examined in the course of dementia research are likely to be among those who would be most at risk, so ongoing vigilance is required.

The lack of in vivo 7T MRI studies investigating neurodegenerative dementias other than $\mathrm{AD}$ and $\mathrm{HD}$ is perhaps surprising, though a reminder is that $7 \mathrm{~T}$ is not widely available as yet. Results from AD studies suggest that $7 \mathrm{~T}$ will be an important method for looking into LBD and FTD also. Like AD, LBD has much of its pathology in small, structurally complex and often peripheral brain areas. Also somewhat surprising is the lack of papers taking a multimodality approach, looking at correlations with PET, EEG or with systemic biological markers (other than APOE) such as immunological markers. Again, multimodal studies are likely to be seen as 7T MRI becomes more widely available.

In summary, although 7T MRI is in its infancy, it shows great promise for neurodegenerative dementia research. Looking to the future, the literature would benefit from larger cohorts, longitudinal data, greater correlation of imaging findings with the clinical picture and research into dementias other than $\mathrm{AD}$ and HD.

Acknowledgements The authors acknowledge the support of the Cambridge NIHR Biomedical Research Centre.

Contributors EFM and JTO conceptualised this systematic review. EFM performed the literature search and reviewed the papers with supervision from JTO. EFM drafted the written paper which was revised by JTO. The authors take joint responsibility for the accuracy and integrity of this work.

Competing interests None declared.

Provenance and peer review Not commissioned; externally peer reviewed.

(c) Article author(s) (or their employer(s) unless otherwise stated in the text of the article) 2017. All rights reserved. No commercial use is permitted unless otherwise expressly granted.

\section{REFERENCES}

1 Prince $M$, Bryce $R$, Albanese $E$, et al. The global prevalence of dementia: a systematic review and metaanalysis. Alzheimers Dement 2013;9:63-75.

2 McKhann G, Drachman D, Folstein M, et al. Clinical diagnosis of Alzheimer's disease: report of the NINCDS-ADRDA Work Group under the auspices of Department of Health and Human Services Task Force on Alzheimer's Disease. Neurology 1984:34:939-44.

3 Braak H, Braak E. Neuropathological stageing of Alzheimer-related changes. Acta Neuropathol 1991;82:239-59. 
4 McKhann GM, Knopman DS, Chertkow H, et al. The diagnosis of dementia' due to Alzheimer's disease: recommendations from the National Institute on AgingAlzheimer's Association workgroups on diagnostic guidelines for Alzheimer's disease. Alzheimers Dement 2011:7:263-9.

5 Jack CR, Lowe VJ, Senjem ML, et al. 11c PiB and structural MRI provide complementary information in imaging of Alzheimer's disease and amnestic mild cognitive impairment. Brain 2008;131:665-80.

6 Hampel H, Bürger K, Teipel SJ, et al. Core candidate neurochemical and imaging biomarkers of Alzheimer's disease. Alzheimers Dement 2008;4:38-48.

7 Zupancic M, Mahajan A, Handa K. Dementia with lewy bodies: diagnosis and management for primary care providers. Prim Care Companion CNS Disord 2011;13.

8 McKeith IG, Dickson DW, Lowe J, et al; Consortium on DLB. Diagnosis and management of dementia with Lewy bodies: third report of the DLB Consortium. Neurology 2005;65:1863-72.

9 Papathanasiou ND, Boutsiadis A, Dickson J, et al. Diagnostic accuracy of ${ }^{123}$ |-FP-CIT (DaTSCAN) in dementia with Lewy bodies: a meta-analysis of published studies. Parkinsonism Relat Disord 2012;18:225-9.

10 Mak E, Su L, Williams GB, et al. Neuroimaging characteristic of dementia with Lewy bodies. Alzheimer's Res Therapy 2014;6:18.

11 Rohrer JD. Structural brain imaging in frontotemporal dementia. Biochim Biophys Acta 2012;1822:325-32.

12 Pringsheim T, Wiltshire K, Day L, et al. The incidence and prevalence of Huntington's disease: a systematic review and meta-analysis. Mov Disord 2012;27:1083-91.

13 Vonsattel JP, DiFiglia M. Huntington disease. J Neuropathol Exp Neurol 1998:57:369-84

14 Niccolini F, Politis M. Neuroimaging in Huntington's disease. World J Radio 2014;6:301-12.

15 Tabrizi SJ, Reilmann R, Roos RA, et al: TRACK-HD Investigators. Potential endpoints for clinical trials in premanifest and early Huntington's disease in the TRACK-HD study: analysis of 24 month observational data. Lancet Neurol 2012;11:42-53.

16 NICE-SCIE Dementia. The NICE-SCIE Guideline on supporting people with dementia and their carers in health and social care. 2007 (updated 2011). http://www.scie. org.uk/publications/misc/dementia/dementia-fullguideline.pdf?res=true (accessed 03 Jun 2016).

17 Chow N, Hwang KS, Hurtz S, et al; Alzheimer's Disease Neuroimaging Initiative. Comparing 3t and 1.5T MRI for mapping hippocampal atrophy in the Alzheimer's Disease Neuroimaging Initiative. AJNR Am J Neuroradiol 2015;36:653-60.

18 Balchandani P, Naidich TP. Ultra-high-field MR neuroimaging. AJNR Am J Neuroradiol 2015:36:1204-15.

19 Moseley ME, Liu C, Rodriguez S, et al. Advances in magnetic resonance neuroimaging. Neurol Clin 2009;27:1-19.

20 Perl DP. Neuropathology of Alzheimer's disease. Mt Sinai J Med 2010;77:32-42.

21 Medicines and Healthcare Products Regulatory Agency. Safety guidelines for magnetic resonance imaging equipment in clinical use. 2015. https://www.gov.uk/ government/uploads/system/uploads/attachment_data/file/476931/MRI_guidance 2015 - 4-02d1.pdf (assessed 02 Jun 2016).

22 Health Protection Agency. Protection of patients and volunteers undergoing RI procedures. Documents of the Health Protection Agency Radiation, Chemical and Environmental Hazards 2008. https://www.gov.uk/government/uploads/system/ uploads/attachment data/file/329364/Protection of patients and volunteers undergoing_MRI_procedures.pdf (accessed 02 Jun 2016).

23 Albert MS, DeKosky ST, Dickson D, et al. The diagnosis of mild cognitive impairment due to Alzheimer's disease: recommendations from the National Institute on Aging-Alzheimer's Association workgroups on diagnostic guidelines for Alzheimer's disease. Alzheimers Dement 2011:7:270-9.

24 Petersen RC, Smith GE, Waring SC, et al. Mild cognitive impairment: clinical characterization and outcome. Arch Neurol 1999:56:303-8.

25 Kerchner GA, Deutsch GK, Zeineh M, et al. Hippocampal CA1 apical neuropil atrophy and memory performance in Alzheimer's disease. Neuroimage 2012:63:194-202.

26 Kerchner GA, Bernstein JD, Fenesy MC, et al. Shared vulnerability of two synapticallyconnected medial temporal lobe areas to age and cognitive decline: a seven tesla magnetic resonance imaging study. J Neurosci 2013;33:16666-72.

27 Kerchner GA, Berdnik D, Shen JC, et al. APOE $\varepsilon 4$ worsens hippocampal CA1 apical neuropil atrophy and episodic memory. Neurology 2014;82:691-7.

28 Wisse LE, Biessels GJ, Heringa SM, et al; Utrecht Vascular Cognitive Impairment (VCI) Study Group. Hippocampal subfield volumes at $7 \mathrm{t}$ in early Alzheimer's disease and normal aging. Neurobiol Aging 2014;35:2039-45.

29 Serrano-Pozo A, Mielke ML, Muzitansky A, et al. Stable size distribution of amyloid plaques over the course of Alzheimer disease. J Neuropathol Exp Neurol 2012:71:694-701.

30 Nakada T, Matsuzawa $H$, Igarashi $H$, et al. In vivo visualization of senile-plaque-like pathology in Alzheimer's disease patients by MR microscopy on a 7T system. J Neuroimaging 2008;18:125-9.

31 van Rooden S, Versluis MJ, Liem MK, et al. Cortical phase changes in Alzheimer's disease at 7T MRI: a novel imaging marker. Alzheimers Dement 2014;10:e19-26.

32 van Rooden S, Doan NT, Versluis MJ, et al. $7 \mathrm{~T} \mathrm{~T}_{2}{ }^{*}$-weighted magnetic resonance imaging reveals cortical phase differences between early- and late-onset Alzheimer's disease. Neurobiol Aging 2015;36:20-6.
33 van Bergen JM, Li X, Hua J, et al. Colocalization of cerebral iron with amyloid beta in mild cognitive Impairment. Sci Rep 2016:6:35514.

34 Brundel M, Heringa SM, de Bresser J, et al. High prevalence of cerebral microbleeds at 7tesla MRI in patients with early Alzheimer's disease. J Alzheimers Dis 2012;31:259-63.

35 Kerchner GA, Hess CP, Hammond-Rosenbluth KE, et al. Hippocampal CA1 apical neuropil atrophy in mild Alzheimer disease visualized with 7-T MRI. Neurology 2010;75:1381-7.

36 Huntington Study Group. Unified Huntington's Diesease Rating Scale: reliability and consistency. Movement Disord Soc 1996;11:136-42.

37 Apple AC, Possin KL, Satris G, et al. Quantitative 7t phase imaging in premanifest Huntington disease. AJNR Am J Neuroradio/ 2014;35:1707-13.

38 van Bergen JM, Hua J, Unschuld PG, et al. Quantitative susceptibility mapping suggests altered brain iron in premanifest Huntington disease. AJNR Am J Neuroradiol 2016:37:789-96.

39 Zhang Y, Long JD, Mills JA, et al; PREDICT-HD Investigators and Coordinators of the Huntington Study Group. Indexing disease progression at study entry with individuals at-risk for Huntington disease. Am J Med Genet B Neuropsychiatr Genet 2011;156B:751-63.

40 Duvernoy HM, Cattin E, Naidich T, et al. The human hippocampus. 3rd edn. Berlin, Heidelberg : Springer-Verlag, 2005.

41 Price JL, Ko Al, Wade MJ, et al. Neuron number in the entorhinal cortex and CA1 in preclinical Alzheimer disease. Arch Neurol 2001;58:1395-402.

42 Frisoni GB, Fox NC, Jack CR, et al. The clinical use of structural MRI in Alzheimer disease. Nat Rev Neurol 2010;6:67-77

43 Wisse LE, Reijmer YD, ter Telgte A, et al; Utrecht Vascular Cognitive Impairment (VCI) Study Group. Hippocampal disconnection in early Alzheimer's disease: a 7 tesla MRI study. J Alzheimers Dis 2015:45:1247-56.

44 Versluis MJ, Peeters JM, van Rooden S, et al. Origin and reduction of motion and fO artifacts in high resolution $\mathrm{T} 2{ }^{*}$-weighted magnetic resonance imaging: application in Alzheimer's disease patients. Neuroimage 2010;51:1082-8.

45 Stucht D, Danishad KA, Schulze $P$, et al. Highest resolution in vivo human brain MRI using prospective motion correction. PLoS One 2015;10:e0133921.

46 Fox NC, Cousens S, Scahill R, et al. Using serial registered brain magnetic resonance imaging to measure disease progression in Alzheimer disease: power calculations and estimates of sample size to detect treatment effects. Arch Neurol 2000;57:339-44.

47 Bertrand E, Lechowicz W, Lewandowska E, et al. Degenerative axonal changes in the hippocampus and amygdala in Parkinson's disease. Folia Neuropathol 2003:41:197-207.

48 Chow N, Aarsland D, Honarpisheh $\mathrm{H}$, et al. Comparing hippocampal atrophy in Alzheimer's dementia and dementia with lewy bodies. Dement Geriatr Cogn Disord 2012;34:44-50

49 Mak E, Su L, Williams GB, et al. Differential atrophy of hippocampal subfields: a comparative study of dementia with Lewy bodies and Alzheimer disease. Am J Geriatr Psychiatry 2016:24:136-43.

50 Deuker L, Doeller CF, Fell J, et al. Human neuroimaging studies on the hippocampal CA3 region - integrating evidence for pattern separation and completion. Front Cell Neurosci 2014;8:64

51 Rabinovici GD, Jagust WJ. Amyloid imaging in aging and dementia: testing the amyloid hypothesis in vivo. Behav Neurol 2009;21:117-28

52 Meadowcroft MD, Connor JR, Smith MB, et al. MRI and histological analysis of betaamyloid plaques in both human Alzheimer's disease and APP/PS1 transgenic mice. J Magn Reson Image 2009;29:997-1007.

53 Zeineh MM, Chen Y, Kitzler HH, et al. Activated iron-containing microglia in the human hippocampus identified by magnetic resonance imaging in Alzheimer disease. Neurobiol Aging 2015:36:2483-500.

54 Connor JR, Menzies SL, St Martin SM, et al. A histochemical study of iron, transferrin, and ferritin in Alzheimer's diseased brains. J Neurosci Res 1992;31:75-83.

55 Sian-Hülsmann J, Mandel S, Youdim MB, et al. The relevance of iron in the pathogenesis of Parkinson's disease. J Neurochem 2011;118:939-57.

56 Gazzina S, Premi E, Zanella I, et al. Iron in frontotemporal lobar degeneration: a new subcortical pathological pathway? Neurodegener Dis 2016;16:172-8.

57 Conijn MM, Geerlings MI, Biessels GJ, et al. Cerebral microbleeds on MR imaging: comparison between 1.5 and 7t. AJNR Am J Neuroradiol 2011:32:1043-9.

58 Cordonnier C, van der Flier WM. Brain microbleeds and Alzheimer's disease: innocent observation or key player? Brain 2011;134:335-44.

59 Brundel M, de Bresser J, van Dillen JJ, et al. Cerebral microinfarcts: a systematic review of neuropathological studies. J Cereb Blood Flow Metab 2012;32:425-36.

60 van Veluw SJ, Heringa SM, Kuijf HJ, et al. Cerebral cortical microinfarcts at 7tesla MRI in patients with early Alzheimer's disease. J Alzheimer's Dis 2014;39:163-7.

61 Muller M, Leavitt BR. Iron dysregulation in Huntington's disease. J Neurochem 2014:130:328-50.

62 Langkammer C, Ropele S, Pirpamer L, et al. MRI for iron mapping in Alzheimer's disease. Neurodegener Dis 2014;13:189-91.

63 Nelson PT, Jicha GA, Kryscio RJ, et al. Low sensitivity in clinical diagnoses of dementia with Lewy bodies. J Neurol 2010;257:359-66. 
64 Calamante F, Oh SH, Tournier JD, et al. Super-resolution track-density imaging of thalamic substructures: comparison with high-resolution anatomical magnetic resonance imaging at 7.0T. Hum Brain Mapp 2013;34:2538-48.

65 Warren JD, Rohrer JD, Rossor MN. Frontotemporal dementia. BMJ 2013;347:f4827.

66 Whitwell JL, Josephs KA. Recent advances in the imaging of frontotemporal dementia. Curr Neurol Neurosci Rep 2012;12:715-23.

67 Boutet C, Chupin M, Lehéricy S, et al. Detection of volume loss in hippocampal layers in Alzheimer's disease using 7 T MRI: a feasibility study. Neuroimage Clin 2014; $5: 341-8$

68 Gregoire SM, Chaudhary UJ, Brown MM, et al. The Microbleed Anatomical Rating Scale (MARS): reliability of a tool to map brain microbleeds. Neurology 2009;73:1759-66.

69 Kuijf HJ, de Bresser J, Geerlings MI, et al. Efficient detection of cerebral microbleeds on 7.0 T MR images using the radial symmetry transform. Neuroimage 2012;59:2266-73.

70 Nakada T. Clinical application of high and ultra high-field MRI. Brain Dev 2007;29:325-35.

71 Li W, Wu B, Liu C. Quantitative susceptibility mapping of human brain reflects spatial variation in tissue composition. Neuroimage 2011:55:1645-56.
72 Cai $K$, Tain R, Das $S$, et al. The feasibility of quantitative MRI of perivascular spaces at 7t. J Neurosci Methods 2015;256:151-6.

73 Hammond KE, Lupo JM, Xu D, et al. Development of a robust method for generating 7.0 T multichannel phase images of the brain with application to normal volunteers and patients with neurological diseases. Neuroimage 2008;39:1682-92.

74 Deistung A, Schäfer A, Schweser F, et al. Toward in vivo histology: a comparison of quantitative susceptibility mapping (QSM) with magnitude-, phase-, and R2* imaging at ultra-high magnetic field strength. Neuroimage 2013:65:299-314.

75 Doan NT, van den Bogaard SJA, Dumas EM, et al. Texture analysis of ultrahigh field T2* -weighted MR images of the brain: application to Huntington's disease. J Magn Reson Image 2014;39:633-40.

76 Chen W, Giger ML, Li H, et al. Volumetric texture analysis of breast lesions on contrast-enhanced magnetic resonance images. Magn Reson Med 2007:58:562-71.

77 Drouin-Ouellet J, Sawiak SJ, Cisbani G, et al. Cerebrovascular and blood-brain barrier impairments in Huntington's disease: potential implications for its pathophysiology. Ann Neurol 2015;78:160-77.

78 Francis ST, Bowtell R, Gowland PA. Modeling and optimization of Look-Locker spin labeling for measuring perfusion and transit time changes in activation studies taking into account arterial blood volume. Magn Reson Med 2008:59:316-25. 